\title{
Diffusive shock acceleration of cosmic rays from two stationary shocks
}

\author{
Masaru Nakanotani ${ }^{1 *}$, Tohru Hada ${ }^{2}$ and Shuichi Matsukiyo ${ }^{3}$
}

\begin{abstract}
Diffusive shock acceleration (Fermi acceleration) of cosmic rays in a system containing two shock waves is investigated using test particle simulations and analysis of the diffusion convection equation. We assume that the cosmic ray acceleration timescale is much less than the approaching timescale of the two shocks. Low-energy cosmic rays are primarily accelerated as they interact with one of the two shocks. However, as they are energized, and their mean free path becomes comparable to the separation distance of the two shocks, they start to accelerate as they interact with both shocks. As a result, a double power-law-type spectrum for the cosmic ray distribution is produced. The numerical result is well explained by a model based on the diffusion convection equation, which accounts for the dependence of the diffusion coefficient on the cosmic ray energy. The break point of the spectrum can be estimated by considering transport of cosmic rays from one shock to the other.
\end{abstract}

Keywords: Cosmic rays, Diffusive shock acceleration, Multiple shocks

\section{Introduction}

One of the most widely accepted mechanisms for producing cosmic rays (energetic particles) is diffusive shock acceleration (DSA). This is also known as first-order Fermi acceleration, in which the cosmic rays gain energy as they are scattered by turbulence upstream and downstream of the shock, repeatedly cross the shock, and are effectively compressed by shock flow Blandford and Eichler (1987). Although the DSA of the cosmic rays has been studied extensively, most past studies have examined the case wherein cosmic rays interact with a single shock wave. The DSA in a system with multiple shocks is not well understood.

Shock waves are ubiquitous in space. For instance, shock waves can be generated in front of coronal mass ejections, in association with the co-rotational interaction regions, and in front of planetary magnetospheres, including that of the earth. These shocks frequently approach and collide with each other. The collision of an interplanetary shock with the earth's bow shock was reported and analyzed in Hietala et al. (2011). The

\footnotetext{
*Correspondence: nakanot@esst.kyushu-u.ac.jp

${ }^{1}$ Interdisciplinary Graduate School of Engineering Sciences, Kyushu

University, 6-1 Kasuga-Koen, Kasuga, Fukuoka 816-8580, Japan

Full list of author information is available at the end of the article
}

authors found that ions were accelerated by first-order Fermi acceleration through the two shocks, and their energy was higher than expected from theory based on the single shock DSA model. When there are two shocks located in close proximity, the DSA model using a single shock may not explain the acceleration process.

Particle acceleration in multiple shocks has been treated theoretically by Melrose and Pope (1993). They considered a system where "seed" particles initially given at the location of the shock waves are continuously accelerated by a sequence of identical shock waves. Furthermore, they concluded that the energy spectrum becomes $n(p) \propto p^{-1}$ for infinite number of shocks, where $n(p)$ is the density distribution function. The spectrum for dual shocks is harder than that of a single shock whose compression ratio is 4. Recently, Zhao and Li (2014) performed test particle simulations for a pair of parallel shocks approaching each other. They included magnetic turbulence and calculated the equation of motion and pitch-angle diffusion of particles. Their calculations showed that the power-law spectrum index for a pair of shocks is harder than that of a single shock, and the maximum energy is greater than that of a single shock.

In the present study, we account for accelerated particles from one shock traveling to the other shock against a plasma flow, an effect that is not considered in Melrose 
and Pope (1993). We discuss the particle acceleration in two shock waves by performing test particle simulations and evaluating the convection diffusion equation.

This paper is organized as follows. In "Introduction" section, we describe our model and assumptions used in our test particle simulation and theoretical analysis. The results of the test particle simulations are presented in "Test particle simulation" section. To explain the simulation results, we develop a theoretical analysis in "Theoretical analysis" section. In "Discussion" section, we discuss the breaking points of the double power-lawtype spectrum by examining the dependence of the break point energy on other parameters. Finally, a summary is provided in "Summary" section.

\section{Test particle simulation}

Let us consider a system in which there are two hydrodynamic planar shocks, "Shock 1" and "Shock 2," located at $x=0$ and $x=L$ in Cartesian coordinates, respectively (Fig. 1). For simplicity, we ignore the background magnetic field. Because we consider this problem as one-dimensional, we do not have to include adiabatic decompression, which is treated in Melrose and Pope (1993). The shocks are assumed to be infinitesimally thin, and both shock normals are pointing toward the $x$ direction. The assumption of zero scale length of the shock transition layer is made due to its simplicity, but it may be violated when a shock precursor with a cosmic ray scattering length scale is generated in front of the shock Drury and Voelk (1981). Here, we simply assume that the energy density of the cosmic rays is small enough.

We further assume that the cosmic ray speed is much faster than the shock speed, and that the timescale of particle acceleration is sufficiently shorter than the timescale for the two shocks to approach each other. Specifically, we assume that shocks are at rest and time stationary. The two shocks divide the entire space into three regions as in Fig. 1: "Region 1" $(x<0)$, "Region 2" $(0<x<L)$,

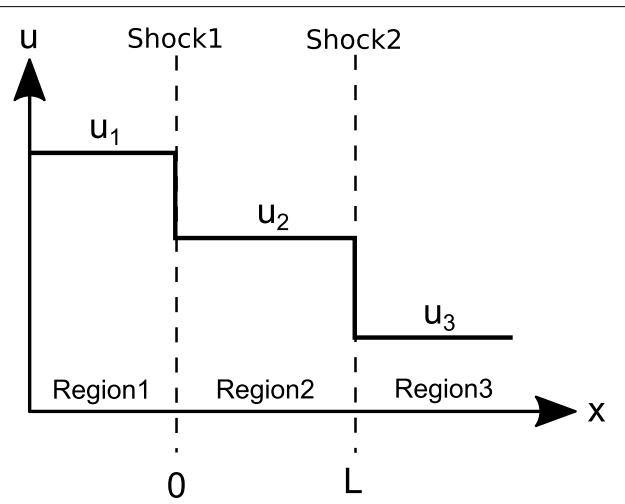

Fig. 1 Schematic showing shock positions, regions, and flow speeds and "Region 3" $(L<x)$, which correspond to upstream of the two shocks, downstream of Shock 1 and upstream of Shock 2 , and downstream of both shocks, respectively.

Next, $\mathbf{p}$ and $\mathbf{p}^{\prime}$ are the cosmic ray momentum vectors defined in the shock rest and plasma rest frames, respectively. These are related through the Lorentz transformation using the local plasma flow velocity, $u(x) \mathbf{e}_{x}$, where $u(x)=u_{i}(=$ const .) for the three regions, $i=1,2,3$, and $\mathbf{e}_{x}$ is the unit vector in the $x$ direction. In the present model, the cosmic ray travels freely with a constant speed along a straight line for the time period, $\tau$, until it collides with a scatterer, i.e., MHD turbulence, which is assumed to move with the local plasma flow.

Because cosmic ray energy is conserved in the plasma rest frame, the cosmic ray momentum after the collision can be written as, $\mathbf{p}_{a}^{\prime}=p^{\prime} \mathbf{e}_{r}$, where $p^{\prime}=\left|\mathbf{p}^{\prime}\right|$ is the magnitude of the momentum in the plasma rest frame, and $\mathbf{e}_{r}$ is a unit vector representing the momentum direction after the collision.

For simplicity, the scattering time, $\tau$, is constant. One can specify $\tau$ according to some given probability distribution function, but within the context of the present study, the generalization for $\tau$ does not alter the results qualitatively, as long as the central limit theorem is satisfied for the convolution of the probability distribution function.

We also assume that cosmic ray scattering due to turbulence is isotropic in the plasma rest frame. ( $\mathbf{e}_{r}$ is chosen randomly in all the directions in three dimensions.) This corresponds to the assumption of large-angle scattering, which may represent the scattering due to a strongly turbulent magnetic field, or an approximation of successive small-angle scattering, such as the pitch-angle scattering by small-amplitude waves. The difference between the large-angle scattering and the pitch-angle scattering models is discussed in Naito and Takahara (1995). We treat the motion of particles in electromagnetic fields as random.

First, we inject seed particles at Shock 1 and Shock 2. The initial particle momentums are all equal to $p_{0}$, but their directions are random. They then travel for $\tau$ and are scattered by turbulence, and a new momentum is computed after collision; they then travel again, and the cycle continues. After repeating this cycle for a certain length of time, we collect particle data and compute the cosmic ray spectrum.

The thick solid line in the large panel shown in Fig. 2 shows the result from the test particle simulation. Test particles are injected at both Shock 1 and Shock 2. Parameters used for this run are: $u_{1}=4, u_{2}=2$, $u_{3}=1$ (normalized to $u_{3}$ ), $L=125$ (normalized to $\tau u_{3}$ ), $p_{0}=\left|\mathbf{p}_{0}\right|=7.5$ (normalized to $m_{0} u_{3}$, where $m_{0}$ is the rest mass of the particle), and the number of particles used is $2 \times 10^{5}$. 
The calculation time is $2 \times 10^{4} \tau$ (normalized to tau). The horizontal axis is the logarithm of the cosmic ray momentum in the shock rest frame, $p$, and the vertical axis is the logarithm of the cosmic ray density distribution function, $n(p)$. Specifically, $n(x, p) \Delta p \Delta x$ corresponds to the number of particles with its position within the range $(x, x+\Delta x)$ and its momentum within the range $(p, p+\Delta p)$. In using these ranges, we ensure that the numerically obtained cosmic ray distribution is nearly isotropic. We collect all the particles in Region 3 to evaluate the distribution function. Because Region 3 corresponds to the downstream of Shock $2, n(x, p)$ must be independent of $x$.

The thick solid line in the small panel in Fig. 2 shows the result of the test particle simulation for one single shock. The conditions are the same with the above simulation except for omitting Shock $2\left(u_{3}=2\right)$ and injecting particles only from Shock 1 . It is well known that the DSA process produces the power-law spectrum, $n(p) \sim p^{-\Gamma}$, with $\Gamma=(r+2) /(r-1)$, where $r$ is the shock compression ratio. For Shock $1, r=u_{1} / u_{2}=2$ and $\Gamma=4$. We confirm that the spectrum obtained from the test particle simulation corresponds to the index from the theoretical analysis.

As shown in the large panel, the spectrum in the lowenergy regime $\left(0<\log \left(p / p_{0}\right)<0.78\right)$ and the highenergy regime $\left(0.78<\log \left(p / p_{0}\right)<2.5\right)$ is approximated by power-law distributions with different indices. The low-energy part is comprised of cosmic rays accelerated by one of the two shocks, and the high-energy part is comprised of particles that travel long enough distances to interact with both shocks. For Shock 1 and Shock 2, $r=u_{1} / u_{2}=u_{2} / u_{3}=2$ and $\Gamma=4$; for the two shocks together the compression ratio is $r=u_{1} / u_{3}=4$, which gives $\Gamma=2$. These values explain the slope of the spectrum for the low- and high-energy regimes in Fig. 2.

\section{Theoretical analysis}

In this section we describe a theoretical model based on the diffusion convection equation (e.g., Skilling 1975). If we assume that the cosmic ray distribution is isotropic, its time evolution can be described by

$$
\frac{\partial f}{\partial t}+u \frac{\partial f}{\partial x}-\frac{p}{3} \frac{\mathrm{d} u}{\mathrm{~d} x} \frac{\partial f}{\partial p}=\frac{\partial}{\partial x}\left(k \frac{\partial f}{\partial x}\right)+Q(x, p, t),
$$

where $f(x, p, t)$ is the cosmic ray distribution function defined in the shock rest frame, $p=|\mathbf{p}|, u(x)$ is the plasma velocity, $k(x)$ is the spatial diffusion coefficient, and $Q(x, p, t)$ is a source term,

$$
Q(x)=A_{1} \delta(x) \delta\left(p-p_{0}\right)+A_{2} \delta(x-L) \delta\left(p-p_{0}\right),
$$

where $A_{1}$ and $A_{2}$ are constant. Within the three regions we defined before, we let $u(x)=u_{i}$ and $k(x)=k_{i}$

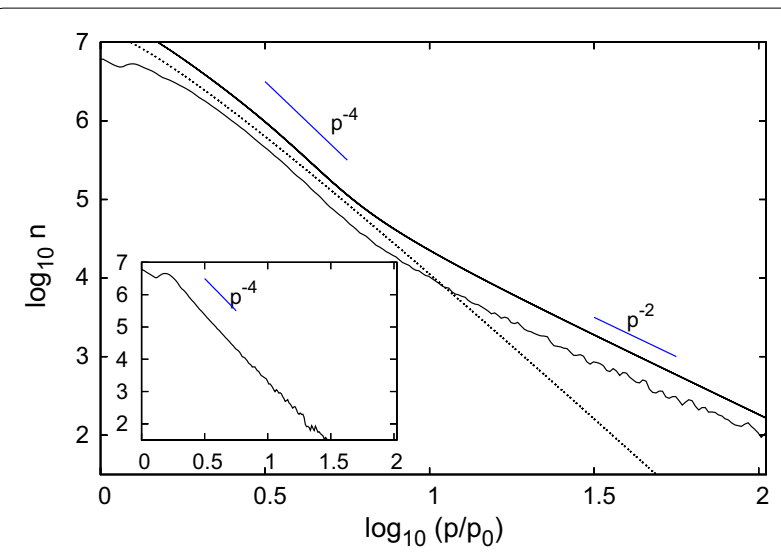

Fig. 2 Large panel: comparison of density distribution obtained from the test particle simulation for two shocks (thick solid line), theoretical model based on the diffusion convection equation with constant diffusion coefficient (dotted line), and the same model that takes into account the dependence of the diffusion coefficient on the cosmic ray energy (thin solid line). The two blue lines with indices -4 and -2 are indicated for reference. Test particles are injected at both

"Shock 1" and "Shock 2." Small panel: density distribution obtained by the test particle simulation for one single shock

( $i=1,2,3)$, with $u_{i}$ and $k_{i}$ constant. Then, the time stationary solution of (1) for each region can be written as

$$
f_{i}(x, p)=\frac{C_{i}(p)}{u_{i}}+B_{i}(p) \exp \left(\frac{u_{i} x}{k_{i}}\right),
$$

where $C_{i}(p)$ and $B_{i}(p)$ are arbitrary functions of $p$. From the boundary conditions at $x \rightarrow \pm \infty$ and continuity of $f_{i}(x, p)$ across the two shocks we find

$$
\begin{gathered}
C_{1}(p)=B_{3}(p)=0, B_{1}(p)=\frac{C_{2}(p)}{u_{2}}+B_{2}(p), \\
\frac{C_{3}(p)}{u_{3}}=\frac{C_{2}(p)}{u_{2}}+B_{2}(p) \exp \left(\frac{u_{2} L}{k_{2}}\right) .
\end{gathered}
$$

Integration of (1) across the first shock at $x=0$ yields

$$
\left[-\left(k \frac{\partial f}{\partial x}+\frac{p}{3} \frac{\partial f}{\partial p} u\right)\right]=0
$$

where the bracket denotes the difference across the shock. Substituting (3) into (5), we have

$$
\begin{aligned}
B_{2} & +\frac{p}{3} \frac{\mathrm{d} B_{2}}{\mathrm{~d} p}+\frac{u_{1}}{u_{1}-u_{2}} \frac{C_{2}}{u_{2}}+\frac{p}{3} \frac{d}{\mathrm{~d} p}\left(\frac{C_{2}}{u_{2}}\right) \\
& =\frac{A_{1}}{u_{1}-u_{2}} \delta\left(p-p_{0}\right) .
\end{aligned}
$$

Similarly, across the second shock at $x=L$, we obtain

$$
\begin{aligned}
& \left(\frac{u_{2} B_{2}}{u_{2}-u_{3}}+\frac{p}{3} \frac{\mathrm{d} B_{2}}{\mathrm{~d} p}+\frac{p B_{2}}{3} \frac{\mathrm{d} \xi}{\mathrm{d} p}\right) e^{\xi}+\frac{p}{3} \frac{\mathrm{d}}{\mathrm{d} p}\left(\frac{C_{2}}{u_{2}}\right) \\
& =\frac{A_{2}}{u_{2}-u_{3}} \delta\left(p-p_{0}\right),
\end{aligned}
$$


where $\xi=u_{2} L / k_{2}(p)$ is a dimensionless parameter representing the relative magnitude of the convection and diffusion for cosmic rays in Region 2. If $\xi>>1$, convection is more dominant than diffusion, and cosmic rays starting from Shock 2 cannot reach Shock 1. Therefore, the two shocks are recognized as two well-separated shocks for particles seeded from Shock 2. On the other hand, if $\xi<<1$, diffusion is more dominant than convection, and the two shocks can essentially be viewed as a single shock with a velocity jump from $u_{1}$ to $u_{3}$.

The value of $\xi$ depends on the momentum of the particle through the spatial diffusion coefficient,

$$
k_{2}=<\frac{\left(v_{x} \tau\right)^{2}}{2 \tau}>=\frac{v^{2} \tau}{6}=\frac{\tau c^{2} p^{2}}{6\left(m_{0}^{2} c^{2}+p^{2}\right)},
$$

where $<>$ denotes the ensemble average, and $v_{x}$ is the $x$ component of the velocity vector in three dimensions. Then (7) is rewritten as

$$
\begin{aligned}
& \left(\left(\frac{u_{2}}{u_{2}-u_{3}}-\frac{2 \xi}{3 \gamma^{2}}\right) B_{2}+\frac{p}{3} \frac{\mathrm{d} B_{2}}{\mathrm{~d} p}\right) e^{\xi}+\frac{p}{3} \frac{d}{\mathrm{~d} p}\left(\frac{C_{2}}{u_{2}}\right) \\
& \quad=\frac{A_{2}}{u_{2}-u_{3}} \delta\left(p-p_{0}\right),
\end{aligned}
$$

where $\gamma=\sqrt{1+\frac{p^{2}}{m_{0}^{2} c^{2}}}$.

Equations (6) and (9) are simultaneous differential equations for $B_{2}(p)$ and $C_{2}(p)$, and the solutions can be obtained numerically. Then, the distribution function is given as

$n(p)=4 \pi p^{2} f_{3}(p)=4 \pi p^{2}\left\{\frac{C_{2}(p)}{u_{2}}+B_{2}(p) \exp \left(\frac{u_{2} L}{k_{2}}\right)\right\}$.

For the DSA with a single shock, the cosmic ray spectrum does not depend on the diffusion coefficient, which is not the case for the present problem. The dotted line in Fig. 2 shows (10), in which $k_{2}$ is given by (8) with $p=p_{0}$. ( $p_{0}$ is the initial momentum.) In this case, $k_{2}$ is a constant. All other parameters are chosen according to the test particle simulation run.

The result does not agree with the simulation. In particular, the model does not have a double power-law distribution produced by the test particle simulation.

The thin solid line in Fig. 2 shows (9) with the $p$ dependence correctly kept in the definition of $k_{2}$ in (8). Now, the result agrees well with the test particle simulation. In particular, the breaking of the power spectrum is included.

To seek an asymptotic solution for (6) and (9), let us write

$$
C_{2}(p)=\alpha p^{-s} ; B_{2}(p)=\beta p^{-s} .
$$

After substituting the above into (6) and (9), and seeking a non-trivial solution for $\alpha$ and $\beta$, we find

$$
s(s-3)=\left(s-s_{1}\right)\left(s-s_{2}\right) e^{\xi},
$$

where $s_{1}=3 u_{1} /\left(u_{1}-u_{2}\right)$ and $s_{2}=3 u_{2} /\left(u_{2}-u_{3}\right)$ are the power-law indices of the density distribution function due to the DSA by Shock 1 and Shock 2, respectively.

In this calculation, we have neglected the second term in (9) for simplicity. When $\xi$ is very large, (12) gives two roots, $s=s_{1}$ and $s=s_{2}$, suggesting that the two shocks are independent. The DSA calculated with the two shocks separately produces distributions specified by their compression ratios. In contrast, in the limit of $\xi \rightarrow 0$, (12) becomes a linear equation for $s$, and the solution is

$$
s=\frac{s_{1} s_{2}}{s_{1}+s_{2}-3}=\frac{3 u_{1}}{u_{1}-u_{3}} .
$$

As expected, the spectrum index from the DSA due to the two shocks combined is obtained. Equation (13) implies that the spectrum of the two shocks can be harder than the strongest single shock. If there are two strong shocks with compression ratios of 4, Eq. (13) becomes 3.2. When considering a series of multiple shocks, the maximum spectrum index can be obtained from Eq. (13), applying the upstream flow of the first shock as $u_{1}$ and the downstream flow of the last shock as $u_{3}$. It is easily seen that the maximum index approaches 3 as the number of shocks increases because the downstream flow of the last shock approaches 0 , in agreement with Melrose and Pope (1993).

\section{Discussion}

We have seen that the diffusion convection equation well explains the test particle simulation results presented in the previous section. In this section, we discuss the dependence of spectrum breaking using Eqs. (6) and (9).

Below we discuss determining the momentum at the breaking point, $p_{b}$, by examining its dependence on shock parameters, $L, u_{1}, u_{2}$, and $u_{3}$.

Figure $3 \mathrm{a}$ shows $p_{b}$ as a function of $L$ (the crosses), keeping other parameters unchanged at $u_{1}=4, u_{2}=2$ and $u_{3}=1$. This plot is made by numerically evaluating the first derivative of $\log n(p)$ with respect to $\log p$. We define the break point as the point at which the first derivative has the minimum value. Figure $3 \mathrm{~b}$ suggests that $p_{b}$ becomes larger as $L$ increases. This relationship is due to the increase in $L$ when a particle starting from Shock 2 must travel a long distance to reach Shock 1.

Figure $3 \mathrm{~b}$ shows $p_{b}$ as $u_{2}$ (circles) varies with parameters $L=125, u_{1}=4$, and $u_{3}=1$. When $u_{2}$ increases, $p_{b}$ also increases, because a particle starting from Shock 2 must overcome the fast convection of the background flow to reach Shock 1. 
The break point is not strongly affected by variations in $u_{1}$ and $u_{3}$ (not shown here) because $u_{1}$ and $u_{3}$ do not influence the particle transport between Shock 1 and Shock 2.

We also estimate the value of $p_{b}$ by comparing the diffusion and convection in Region 2. Suppose a particle with momentum $p$, located at Shock 2, starts a random walk at time $t=0$ in a convecting flow with a speed $u_{2}$. The motion of the particle may then be given as a superposition of the random walk and convection,

$$
\lambda(t)=\sqrt{6 k_{2} t}-u_{2} t
$$

where $\lambda$ is the travel distance along the $x$ direction measured from the location of Shock 2 at $x=L$. Because $k_{2}$ is an increasing function of the particle momentum $p$, faster particles can travel longer distances compared with slower particles. Moreover, due to convection, there is a maximum distance a particle can travel, $\lambda_{\max }=3 k_{2} / 2 u_{2}$. We estimate that the momentum at which $\lambda_{\max }=L$ gives $p_{b}$. Namely, particles with momentum larger than $p_{b}$ can travel from one shock to the other, potentially accelerating from both shocks. From this argument, we estimate

$$
\frac{p_{b}}{m_{0} u_{3}}=\sqrt{\frac{\delta}{1-\delta\left(\frac{u_{3}}{c}\right)^{2}}},
$$

where $\delta=4\left(L / \tau u_{3}\right)\left(u_{2} / u_{3}\right)$ and $c$ is the speed of light.

In Fig. 3 we plot (15) as the solid line for $L$ (a) and the dotted line for $u_{2}$ (b). This model well explains the functional dependence of $p_{b}$ on $L$ and $u_{2}$. This dependence of $p_{b}$ on $L$ becomes different between simulation and theoretical results for large $L$ because we implicitly assume that the distribution function can spread out over the distance $\lambda_{\max }$, which a particle can reach when we consider time stationary solution (4).
The spatial diffusion coefficient approaches $\tau c^{2} / 6$ for highly relativistic particles. In this case, $\lambda_{\max }$ is written as $\left(c / 4 u_{2}\right) \tau c$. Therefore, considering the time stationary solution, the condition $L<<\left(c / 4 u_{2}\right) \tau c$ should be applied.

\section{Summary}

In summary, we examined the DSA process in a system including two shocks. We assume that an acceleration timescale in a shock is much less than the approaching timescale of the two shocks. These two shocks can be treated as stationary under this assumption. Although we have to consider finite acceleration time for a more realist case, which means two shocks moving at different speeds, we believe that it does not change the result significantly.

Test particle simulations are performed, and a double power-law-type spectrum is obtained. A simple model based on the diffusion convection equation explains the result well. In the model, it is important to include the momentum dependence on the spatial diffusion coefficient, unlike the DSA model with a single shock.

We discussed the dependence of the break point, $p_{b}$, on other shock parameters, and showed that the value of $p_{b}$ can be estimated by balancing the convection and diffusion in a region between the two shocks. Our results indicate that an acceleration in two shocks is more efficient than a single shock, which is consistent with previous results.

The DSA in a two-shock system is similar to the DSA from cosmic ray-mediated shocks, in that the background flow velocity difference used to accelerate particles depends on particle energy, which forms the double power-law spectrum. Similarly, spectral hardening has been observed at solar flare termination shocks Li et al.
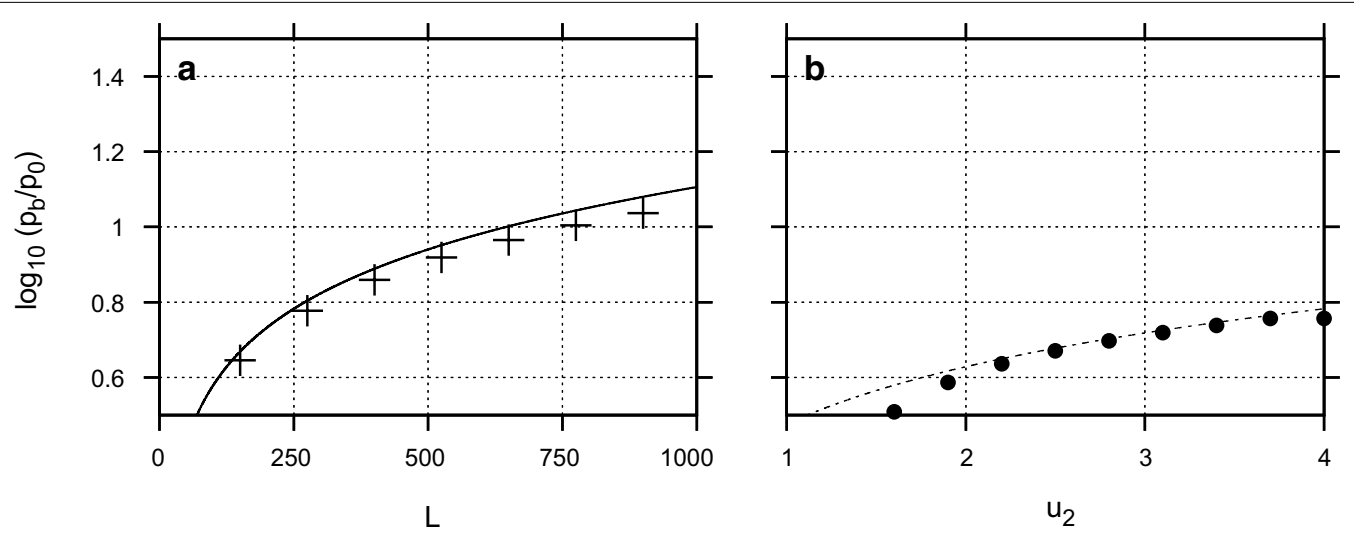

Fig. 3 The breaking point $p_{b}$ as a function of $L$ (crosses) and $u_{2}$ (circles), numerically evaluated using the first derivative of log $n(p)$ with respect to $\log p$. Solid lines are Eq. (15) 
(2013) and Kong et al. (2013). In these previous studies, the finite thickness of the termination shock was considered, and it was concluded that spectral hardening occurs because high-energy electrons can see the full width, while low-energy particles accelerate for only part of the shock width.

\section{Authors' contributions}

MN designed the test particle simulation algorithm and drafted the manuscript. TH and SM helped analyze and interpret the results and draft the manuscript. All authors read and approved the final manuscript.

\section{Author details}

1 Interdisciplinary Graduate School of Engineering Sciences, Kyushu University 6-1 Kasuga-Koen, Kasuga, Fukuoka 816-8580, Japan. ${ }^{2}$ Faculty of Engineering Sciences, Kyushu University, 6-1 Kasuga-Koen, Kasuga, Fukuoka 816-8580, Japan. ${ }^{3}$ International Center for Space Weather Science and Education, Kyushu University, 744 Motooka, Nishi-Ku, Fukuoka 819-0395, Japan.

\section{Acknowlegements}

We thank C. Mazelle for carefully reading the manuscript.

\section{Competing interests}

The authors declare that they have no competing interests.

Ethics approval and consent to participate

Not applicable.

\section{Publisher's Note}

Springer Nature remains neutral with regard to jurisdictional claims in published maps and institutional affiliations.

Received: 8 November 2017 Accepted: 6 February 2018

Published online: 21 February 2018
References

Blandford R, Eichler D (1987) Particle acceleration at astrophysical shocks: a theory of cosmic ray origin. Phys Rep 154:1-75

Drury LO, Voelk JH (1981) Hydromagnetic shock structure in the presence of cosmic rays. Astrophys J 248:344-351

Hietala H, Agueda N, AndréEová K, Vainio R, Nylund S, Kilpua EKJ, Koskinen HEJ (2011) In situ observations of particle acceleration in shock-shock interaction. J Geophys Res (Space Physics) 116:10105

Kong X, Li G, Chen Y (2013) A statistical study of the spectral hardening of continuum emission in solar flares. Astrophys J 774:140

Li G, Kong X, Zank G, Chen Y (2013) On the spectral hardening at GSIM 300 kev in solar flares. Astrophys J 769:22

Melrose DB, Pope MH (1993) Diffusive shock acceleration by multiple shocks. Proc Astronom Soc Aust 10:222

Naito T, Takahara F (1995) Monte carlo simulation of diffusive particle acceleration in shock waves with oblique magnetic fields. Mon Not R Astronom Soc 275:1077-1092

Skilling J (1975) Cosmic ray streaming. I_effect of alfven waves on particles. Mon Not R Astronom Soc 172:557-566

Zhao L, Li G (2014) Particle acceleration at a pair of parallel shocks near the sun. J Geophys Res (Space Physics) 119:6106-6119

\section{Submit your manuscript to a SpringerOpen ${ }^{\circ}$ journal and benefit from:}

- Convenient online submission

- Rigorous peer review

- Open access: articles freely available online

- High visibility within the field

- Retaining the copyright to your article

Submit your next manuscript at $\boldsymbol{\nabla}$ springeropen.com 